\title{
Informationskompetenz
}

\section{Claus Harringer* \\ „Astroturfing“ als Desinformationsstrategie am Beispiel (heimlich) pharmafinanzierter Patientinnen-Initiativen}

https://doi.org/10.1515/iwp-2020-2129

Desinformationskampagnen können verschiedene Formen annehmen, bzw. Strategien verfolgen: Etwa, Konfusionen erzeugen, um relevante Tatsachen mit Nebensächlichkeiten zu überdecken. Oder es wird durch propagandistische Anstrengungen ein eigenes Narrativ, eine Gegenerzählung etabliert, um den wissenschaftlichen Konsens in Zweifel zu ziehen, weil er konkreten Interessen widerspricht. Ein z. B. bei Tabak- Öl- und Chemiekonzernen beliebtes Vorgehen besteht darin, durch Verweis auf „abweichende fachliche Positionen“ Zweifel an etablierten Erkenntnissen zu streuen (Oreskes, Conway 2010). Diese Positionen basieren dabei oft auf Studien, die von den Konzernen selbst in Auftrag gegeben und finanziert werden. Wenn Firmen aber zu offensiv Studien sponsern und Forschende protegieren, ist das kontraproduktiv, da deren Glaubwürdigkeit leidet. Als beste Maßnahme gegen Desinformationskampagnen wird meist die möglichst kritische Prüfung der Quellen empfohlen (neudeutsch „Faktencheck“ genannt), um so glaubwürdige von unglaubwürdigen Informationen $\mathrm{zu}$ unterscheiden. Im medizinischen Bereich ist das z.B. deshalb möglich, weil durchgesetzt wurde, dass potentielle Interessenkonflikte (Conflicts of interest) tatsächlich ausgewiesen werden. Ich möchte im Folgenden argumentieren, dass dieser Ratschlag im Falle von Astroturfing nicht greift, weil oft genau jene Informationen fehlen, mittels derer wesentliche Zusammenhänge hergestellt werden können. Dazu werde ich zunächst skizzieren, was unter dem Begriff „Astroturfing“ $z u$ verstehen ist (1) und einige Beispiele anführen,

Anmerkung: Dieser Artikel basiert auf einem Audiobeitrag für das virtuelle Symposion „Aufzeigen! Initiativen Petitionen NGOs“ des Kulturinstituts an der Johannes Kepler Universität. Nachzuhören unter http://www.kulturinstitut.jku.at/symposion2020.html [10.11.2020].

*Kontaktperson: Claus Harringer, B. A., M. A., Johannes Kepler Universität Linz, Institut für Philosophie und Wissenschaftstheorie, Altenberger Straße 69, 4040 Linz, E-Mail: claus.harringer@jku.at, https://orcid.org/0000-0001-7697-1311 um das konkrete Vorgehen dabei zu illustrieren (2). Anschließend gehe ich auf die Dimension des Phänomens ein (3) und führe abschließend einige Vorschläge an, wie dieser Praxis entgegenzutreten wäre (4).

\section{Astroturfing: Was hat Kunstrasen mit Graswurzeln zu tun?}

„Astroturf“ ist ein US-amerikanischer Markenname für Kunstrasen, der seit den 1960er Jahren patentiert ist. Mittlerweile steht „Astroturf“ für Kunstrasen insgesamt. „Astroturfing“ bedeutet die künstliche Nachahmung von Graswurzelbewegungen (,grassroot movements"). Das vom Verein LobbyControl betriebenen Onlinelexikon Lobbypedia definiert Astroturfing als „das künstliche Nachahmen einer Bürgerbewegung, die hinter den Kulissen von Unternehmen oder Lobbyorganisationen gesteuert oder finanziert wird. Die Tarnung soll den Geldgebern dazu dienen, von der besonderen Glaubwürdigkeit von Bürgerinitiativen zu profitieren. ${ }^{\text {11 }}$ Bei Initiativen der Bevölkerung geht man gemeinhin davon aus, dass finanzieller Gewinn nicht das ausschlaggebende Motiv ist, sondern Hingabe an die jeweilige Sache. Genau das möchten Firmen für sich reklamieren, um glaubwürdig zu erscheinen. Dass Pharmafirmen daran teilhaben möchten, ist nachvollziehbar rangieren deren Sympathiewerte aufgrund ihrer Geschäftspraktiken doch irgendwo zwischen Erdölunternehmen, Tabakkonzernen, Waffenherstellern oder Drogenkartellen. Astroturfing funktioniert umso besser, je weniger über die tatsächliche Beteiligung der Pharmafirmen an den Patientinnen-Initiativen ${ }^{2}$ bekannt ist. Darin besteht aber auch gerade das informationsethische Problem, da

1 https://lobbypedia.de/wiki/Astroturfing [7.9.2020].

2 Es wurde soweit möglich eine gendergerechte Ausdrucksweise und nur in Einzelfällen für die bessere Lesbarkeit des Textes das generische Femininum verwendet, gemeint sind jedoch immer alle Geschlechter. 
die Unterscheidung von relevanten Informationen und Marketing unter diesen Voraussetzungen nicht getroffen werden kann.

\section{Fallstudien}

Die folgenden Beispiele zeigen, wie Astroturfing konkret abläuft und welche Strategien dabei zur Anwendung kommen.

\section{Pain Care Forum}

Die missbräuchliche Verschreibung von Schmerzmitteln auf Opioid-Basis hat in den USA zu einer Situation geführt, die als „Opioid-Epidemie“ bezeichnet wird. Nach Angaben des Center for Disease Control and Prevention (CDC) starben 2018 in den USA über 67.000 Menschen an einer Überdosis Drogen - bei 70 Prozent der Todesfälle waren Opioide die Ursache: Sowohl illegal wie auch legal erworbene. ${ }^{3}$ Die Grenzen sind fließend, da viele Suchterkrankungen mit der Verschreibung von Opioid-Schmerzmitteln beginnen. Es ist nicht leicht zu sagen, ob Pharmafirmen oder Drogenkartelle das größere Leid verursachten - letztere übernahmen nach massiven Produktionserweitungen und Preissenkungen diejenigen als „Kundschaft“, die aufgrund der Praktiken ersterer süchtig geworden waren (Sismondo 2018). Eine besonders unrühmliche Rolle nahm der Pharmakonzern Purdue mit seinem Medikament OxyContin ein, das 1996 auf den Markt kam. Purdue bestritt, dass OxyContin die Opioid-Epidemie maßgeblich mitverursacht hat. 2019 wurden Dokumente publik, die zeigen, dass Purdue und andere sehr gezielt eine künstliche Graswurzelbewegung orchestriert haben. Richard Sackler, der ehemalige Präsident von Purdue, bezeichnete diese als „pain movement“ (Lurie 2019). Diese Dokumente stammen aus einem Rechtsstreit vor US-amerikanischen Bundesgerichten, bei dem 2.000 Klagen gegen Opioid-Hersteller gebündelt verhandelt wurden. Die Konzerne Purdue und Johnson \& Johnson (eigentlich Konkurrenten) finanzierten Organisationen wie die American Pain Foundation, die American Pain Society, die U.S. Pain Foundation und die American Academy of Pain Medicine. Deren Sprachrohre traten in Fernsehtalkshows und bei Konferenzen auf und versuchten, Ärztinnen und Ärzte durch medizinische Fortbildungen von den Schmerzmitteln zu überzeugen. Die U.S. Pain Foundation

3 https://www.cdc.gov/drugoverdose/data/statedeaths.html [13.9. 2020]. hat insgesamt beinahe drei Millionen US-Dollar erhalten (Horwitz et al. 2019). Deren „spokes persons“ entwickelten zusammen mit den Pharmakonzernen Informationsbroschüren, die an Krankenhäuser verschickt wurden und platzierten wöchentlich Artikel über Schmerzbekämpfung in Zeitungen. Einige Gruppen veröffentlichten Richtlinien, die das Risiko einer Opioid-Abhängigkeit herunterspielten oder lobbyierten für die Änderung von Gesetzen, die Opioid-Missbrauch eindämmen sollten und unterstützten auch Ärztinnen und Ärzte, die wegen der Überverschreibung von Schmerzmitteln verklagt worden waren. Gerichtsakten zufolge schlossen Purdue und Abbott Laboratories 1996 ein „co-promotion agreement“ zur Bewerbung von OxyContin ab (U.S. GAO 2003) und 2000 einigten sich Purdue und Johnson \& Johnson in einer analogen Vereinbarung darauf, ihre Opioid-Produkte wechselseitig über ihre Handelsvertretende zu bewerben (Lurie 2019). Solche informellen Absprachen sind Ansätze zur Kartellbildung. Als 2001 erste Medienberichte über weit verbreiteten Missbrauch von OxyContin auftauchten, wandte sich Dr.in Kathleen Foley, Neurologin an einem New Yorker Krebszentrum und ehemals bezahlte Sprecherin für Purdue via Email an Richard Sackler und drängte ihn, mit anderen Pharmafirmen zu kollaborieren. 2005 wurde dann das „Pain Care Forum“ organisiert - unter seinen ersten Teilnehmenden waren Johnson \& Johnson, Purdue und die American Pain Foundation. Gerichtsdokumenten zufolge beteiligte sich im Laufe der Zeit aber so ziemlich jeder größere Opioid-Hersteller und Vertrieb in den USA. Das Forum hatte weder eine Mitgliederliste, noch eine öffentliche Website - es war eine informelle Plattform für Diskussion und zur Koordinierung medialer und politischer Kampagnen. Diese wurden nötig, als das CDC 2005 Richtlinien vorschlug, die die ärztliche Verschreibung von Opioiden einschränken sollten. Dagegen wurden Kampagnen kreiert, die den Eindruck von Graswurzelbewegungen erwecken sollten - gerade auch durch die Rekrutierung von Betroffenen. Die Mitglieder des Pain Care Forum gaben zwischen 2006 und 2015 für die Beeinflussung der Regierung rund 40 Millionen US-Dollar aus (Perrone et al. 2016). Purdue wurde für seine Mitschuld an der Opioid-Krise 2019 zu Entschädigungszahlungen in Höhe von 12 Milliarden US-Dollar verurteilt, meldete daraufhin Insolvenz an und einigte sich mit den Behörden letztlich auf einen Vergleich.

\section{Cancer United}

2006 wurde in Brüssel die Initiative Cancer United gegründet - ein Zusammenschluss aus Betroffenenverbänden und Personen aus der Medizin, Wissenschaft, Politik und 
Industrie. Als Ziel wurde formuliert, die Krebsbehandlungen europaweit anzugleichen. Die britische Zeitung „The Guardian" berichtete einige Stunden vor dem feierlichen Start von Cancer United am 18. Oktober 2006, dass diese paneuropäische Krebskampagne zur Gänze vom Pharmariesen Roche finanziert wurde (Boseley 2006). Mit Herceptin und Avastin hat Roche zwei Antikörper-Wirkstoffe im Programm, die bei Brustkrebs, bzw. auch anderen fortgeschrittenen Krebserkrankungen zur Anwendung kommen. Das Sekretariat von Cancer United wurde praktischerweise von der PR-Firma Weber Shandwick besetzt, die von Roche mit der Kampagne beauftragt worden war und eine Roche-Führungskraft saß im Vorstand. Cancer United bzw. Weber Shandwick bewarben Herceptin und Avastin unter der Ärzteschaft und der Presse - die Studie, auf die sie sich dabei stützten, war ebenfalls von Roche finanziert worden. Aus dem Grund zogen sich auch einige Personen aus dem Vorstand von Cancer United zurück andererseits kooptierte der Verein Personen, die gar nichts davon wussten, wie z.B. Lynn Faulds Wood, die Vorsitzende der European Cancer Patients Coalition. Sie forderte die Vereinigung auf, sie aus dem Vorstand zu entfernen (ebd.). Auch sonst gab es an der Kampagne vereinzelt Kritik von Betroffenenverbänden und aus der Ärzteschaft, die sie als Marketingschachzug von Roche bezeichneten. Dennoch existiert Cancer United noch heute.

\section{Ausmaß}

Astroturfing ist ein globales Phänomen, aber leider nicht als solches erforscht, was die Geldflüsse angeht. Ich konzentriere mich deshalb auf Studien zur Situation in den USA und der EU, sowie Großbritannien und Österreich.

\section{USA}

Der Datendienstleister Bloomberg Government gibt an, dass sechs Pharmakonzerne (namentlich AbbVie Inc., Bristol-Myers Squibb Co., Pfizer Inc., Merck \& Co. Inc., AstraZeneca PLC und Johnson \& Johnson) im Jahr 2018 zusammen in den USA 680 Millionen Dollar an hunderte Nonprofit-Organisationen verteilt haben (Ruoff 2019). Damit haben sich die Ausgaben für Unterstützungsgruppen für Erkrankte und Non-Profits in den drei Jahren nach 2015 mehr als verdoppelt - in dem Jahr waren es noch 321 Millionen Dollar. Die Daten dazu stammen aus den Steuererklärungen der Konzerne, sowie aus Unterlagen, die 2019 dem Finanzausschuss des US-Senats übermittelt worden waren. Ein Teil der Gelder wurde auch für Ausbil- dungsförderungen und Stipendienprogramme für Ärztinnen und Ärzte ausgegeben. Interessant ist vor allem die Relation zu anderen Finanzposten: Die 680 Millionen Dollar, die die sechs Konzerne 2018 für Non-Profit- und Patientinnen-Organisationen ausgegeben haben, übertreffen bei weitem die Aufwendungen für direktes Lobbying, um die Preispolitik im eigenen Sinn zu beeinflussen. Diejenige Non-Profit-Vereinigung, die in den letzten Jahren das meiste Geld bekam, ist die Patient Access Network Foundation (ebd.). Die Konzerne versprechen sich von der Respektabilität und Authentizität solcher Gruppierungen also mehr als von direkten Beeinflussungsversuchen. Dabei bemühen sowohl die Organisationen als auch die Konzerne die Schutzbehauptung, dass es weniger um Einflussnahme gehe, als um den Zugang zu Fachwissen (ebd.). Dass manche „patient advocacy groups“, die von den sechs erwähnten Pharmakonzernen Millionenbeträge erhalten haben, gegen Pläne mobil machten, nach denen Ärztinnen und Ärzte zuerst Medikamente verschreiben sollten, die von den Krankenversicherern festgelegt werden (meist Generika), bevor sie zu teureren Präparaten wechseln, stimmt bequemerweise mit den Interessen der Pharmakonzerne überein. Damit läuft eine „patient advocacy group“ aber Gefahr, zum „Advocatus diaboli“ zu werden. Die von der Kaiser Family Foundation betriebene Datenbank „Pre\$cription For Power“ enthält über 1.200 „patient advocacy groups“ von denen mehr als die Hälfte $(n=650)$ nachweislich Geld von Pharmafirmen bekommen hat. ${ }^{4}$ Das herauszufinden ist auch in den USA nicht besonders leicht, obwohl dort größere Informationsfreiheit herrscht. Als einkommenssteuerbefreite Non-Profit-Organisationen müssen Patientinnen-Vertretungen ihre Spenderinnen und Spender von Beträgen über 5.000 US-Dollar seit Juli 2018 nicht mehr offenlegen (Lorenzo 2018). Bis dahin gab es die Verpflichtung, Informationen zu Spenden über 5.000 Dollar nicht nur im entsprechenden Formular $990 \mathrm{zu}$ vermerken, sondern dieses auch öffentlich zugänglich zu machen. Gesammelt werden, müssen die Informationen zu den Spendenden aber weiterhin, um sie auf Anfrage dem US-Bundesfinanzamt IRS zur Verfügung zu stellen. Begründet wurde die Änderung mit der vereinfachten Steuerverwaltung und dem besseren Schutz von Spendendenden (ebd.). Eine 2017 im New England Journal of Medicine erschienene Studie untersuchte die Spendenauskunft von 104 US-amerikanischen ,patient advocacy groups“ und kam zu dem Ergebnis, dass zwar fast 90 Prozent die Namen ihrer Spenderinnen und Spender veröffentlichten, aber nur ca. 60 Prozent die Höhe der Spen-

4 https://khn.org/patient-advocacy/\#all-organizations [13.9.2020]. 
den. Nur bei 5 Prozent waren diese Angaben in Form exakter Zahlen verfügbar (McCoy et al. 2017).

\section{Europäische Union}

Für Europa ist es noch schwieriger, an Zahlen zu kommen: Als Dachorganisation für Patientinnen-Initiativen fungiert das European Patients Forum (EPF). Es vertritt derzeit 74 Mitglieder - sowohl paneuropäische Plattformen als auch nationale Organisationen. Anhand der Mitgliederliste kann man die einzelnen Organisationen durchgehen und nach Informationen über deren Finanzierung suchen. Dies ist allerdings nicht besonders ergiebig - im besten Fall sind Partner und Sponsoren gelistet, darunter - wenig überraschend - häufig Pharmafirmen. Über die Art der Unterstützung oder gar die Höhe finanzieller Zuwendungen findet man wenig. Das seit 2011 bestehende europäische Transparenz-Register ist nur bedingt hilfreich - dort erfährt man zwar, dass mit Ende April 202011.623 Organisationen registriert waren und in welche groben Kategorien sie fallen (Beratungsfirmen, In-House Lobbyorganisationen, Universitäten, Nichtregierungsorganisationen, Wirtschaftsverbände, etc.) Darüber hinaus wird es aber unübersichtlich und damit aufwendig. Eine Möglichkeit ist, die Liste der EPF-Mitglieder einzeln durchzugehen und im Transparenzregister nach Informationen über sie zu suchen. Befriedigend ist das nicht. Zwar gibt es das europäische Spendensiegel, doch ist dies keine offizielle, sondern eine private Initiative. Das Deutsche Zentralinstitut für soziale Fragen (DZI) hat außerdem 2012 in einer Stellungnahme darauf hingewiesen, dass der Initiator des europäischen Spendensiegels zugleich Betreiber eines Beratungsunternehmen für Vereine und Stiftungen ist. Der Verdacht, dass es sich dabei eher um eine PR-Aktion handelt, ist naheliegend - ein offizielles europäisches Spendensiegel gibt es jedenfalls nicht (DZI 2012). Daneben existieren noch unverbindliche Richtlinien, die aber von der Pharmaindustrie selbst ausgearbeitet wurden: Die European Federation of Pharmaceutical Industries and Associations (EFPIA) hat im Jahr 2017 ein Papier mit dem Titel „Working Together with Patient Groups“ präsentiert, an dem laut Selbstauskunft neben Mitgliedern der Europäischen Pharmaföderation auch Patientinnen-Organisationen beteiligt waren. Strenge Selbstverpflichtungen finden sich darin keine - die sollte man von einem Zusammenschluss auch nicht erwarten, dessen erklärtes Ziel die Steigerung der Konkurrenzfähigkeit der europäischen Pharmafirmen ist. Das am Papier beteiligte European Patients' Forum stellt dafür auf seiner Website ein „Fundraising Toolkit for Patients Organisations“ zu Verfügung: Unter „Ethics, Trans- parancy, Accountability“ heißt es, dass die Accountability am besten durch regelmäßige Berichte zu erreichen sei. Im Unterschied zu Firmen seien diese aber nicht verpflichtend (Biancu et al. 2016). Allerdings können Patientinnen-Organisationen nicht verhindern, dass Zahlungen an sie in den Berichten der zahlenden Firmen auftauchen. Die EFPIA verlangt nämlich von allen ihren Mitgliedern, ihrem „disclosure code“ $z u$ entsprechen und derartige Zahlungen zu veröffentlichen. Dennoch sind die Geldflüsse von Pharmafirmen an Betroffenen-Organisationen europaweit schwer nachvollziehbar. Ein Grund dafür ist die fehlende Verpflichtung zur Offenlegung von Spenden. Dankenswerterweise gibt es einzelne Initiativen, die sich trotzdem die Mühe machen, die finanziellen Verflechtungen $\mathrm{zu}$ untersuchen, etwa die Niederländische NGO Health Action International: Sie veröffentlichte 2011 zusammen mit der Universität Groningen einen Forschungsartikel unter dem Titel „The patient \& consumer voice and pharmaceutical industry sponsorship“ (Perehudoff/Alves 2011). Dafür verschickten die beiden Autorinnen strukturierte Fragebögen an 22 europäische Patientinnen- und Konsumentinnen-Organisationen und analysierten deren veröffentlichte Strategiepapiere sowie die zugänglichen Finanzdaten. Die Autorinnen halten klar fest, dass dieses methodische Vorgehen die Sponsoring-Realität eventuell nicht exakt trifft, es aufgrund fehlender einheitlicher Regeln für die Offenlegung von Spenden aber kaum eine Alternative dazu gab. Sie kommen zum Schluss, dass der Erhalt von Unternehmenssponsoring die Bereitschaft erhöht, Industrieinformationen größeren Raum zu geben (ebd.).

\section{Großbritannien}

2019 erschien im British Medical Journal eine Analyse der Zahlungen von Pharmafirmen an Patientinnen-Organisationen in Großbritannien. Diese kam zum Ergebnis, dass zwischen 2012 und 2016 die Summe von 57 Millionen Pfund (65 Millionen Euro) geflossen ist, wobei sich die jährliche Summe über den untersuchten Zeitraum verdoppelt hat. Die finanzielle Unterstützung ging gezielt an einige wenige Organisationen - vorrangig an solche, die sich mit kommerziell interessanten Krankheiten befassen. Für die Analyse wurden (Ozieranski et al. 2019) auf Konzern-Webseiten nach „disclosure documents“ gesucht - sowohl bei den Mitgliedern des europäischen Dachverbands EFPIA, als auch dem Pendant auf nationaler Ebene in Großbritannien: der Association of the British Pharmaceutical Industry (ABPI). Die ABPI hat für ihre Mitglieder eine Offenlegungsverpflichtung analog zum „disclosure code“ der EFPIA eingeführt - diese nennt sich 
„disclosure UK“ und verfügt zumindest über eine Datenbank ${ }^{5} .2016$ veröffentlichten ca. zwei Drittel der ABPI-Mitgliedsunternehmen ( $\mathrm{n}=35$ von $\mathrm{n}=53$ ) Berichte über Zahlungen an Patientinnen-Organisationen. Das Autorenteam hält fest, dass es den Offenlegungen an Transparenz mangele, wie so oft wenn die Industrie sich selbst reguliert. Die erwähnten 57 Millionen Pfund zwischen 2012 und 2016 teilen sich auf ca. 4.500 offengelegten Einzelzahlungen auf. Verglichen mit den Geldern an Gesundheitspersonal und Gesundheitsorganisationen ist das wenig: Die bekamen von der britischen Pharmaindustrie allein im Jahr 2015 mit 340 Millionen Pfund sechs Mal so viel. Am häufigsten ging es um kleinere Summen (unter 5.000 Pfund/Jahr) - die sollten aber nicht unterschätzt werden. Studien konnten zeigen, dass auch sie das Verschreibungsverhalten von Ärztinnen und Ärzten verändern (Fleischman et al. 2016). Bei den Zahlungen dominierte Big Pharma - fast 70 Prozent stammten von den 10 größten Unternehmen. Das meiste Geld (über 47 Millionen Pfund) ging an jene 314 der 508 Organisationen, die mehrere Ziele verfolgten: Interessenvertretung von Erkrankten, politische Beteiligung, Bewusstseinsbildung oder auch Forschung. Organisationen, die sich nur auf eine oder zwei Aktivitäten konzentrierten (Unterstützung oder Repräsentation), bekamen weniger Geld. Von oberster Förderpriorität waren für die Industrie besonders jene Aktivitäten, die sich an Publikum außerhalb des Betroffenenkreises richteten. Die Unterstützung von Erkrankten oder Aufrechterhaltung der Organisation waren für die Pharmaindustrie weniger förderwürdig (Ozieranski et al. 2019). Das deutet nach Ozieranski et al. 2019 zum einen auf eine Professionalisierung der Organisationen hin, bei der das Engagement nach Außen einen Schlüsselbereich darstellt. Andererseits zeigt es auch, dass Betroffene für die Industrie vermehrt zum Hebel werden, um ihren Einfluss in relevanten Feldern wie Auftragsvergabe, Entwicklungsvorschriften und Zulassung zu erhöhen. Bei den Krankheitsgruppen lag die monetäre Priorität klar bei Krebserkrankungen - über ein Drittel der Finanzmittel gingen an diesen Bereich, gefolgt von hormonellen und Stoffwechselerkrankungen (11\%). Für Infektions- und parasitische Erkrankungen waren es 8 Prozent. Dabei ist bemerkenswert (aber wenig überraschend), dass die größten Spender gerade die Unternehmen waren, die in dem Zeitraum, bzw. danach teure Medikamente für die entsprechenden Krankheiten auf den Markt brachten. Die britischen Ergebnisse zeigen, dass Unternehmen nicht einmal gezielt auf die Inhalte Einfluss nehmen müssen, die sie

5 https://search.disclosureuk.org.uk/ [13.9.2020]. sponsern. Allein durch ihre finanziellen Unterstützungen der außenwirksamen Aktivitäten formen sie bereits die Profile der Organisationen - was wiederum die Wahrnehmung der politischen Entscheidungstragenden beeinflusst, bzw. auch die der verschreibenden Ärzteschaft. Durch die Konzentration auf bestimmte Organisationen, Aktivitäten und Krankheiten verstärkt die Industrie Ungleichverteilungen zwischen Organisationen - besonders vor dem Hintergrund einer schwachen Förderung durch die öffentliche Hand. Die genauen Mechanismen zu untersuchen werde aber dadurch erschwert, dass die finanziellen Verbindungen zwischen Industrie und PatientinnenOrganisationen im Dunkeln liegen (ebd.).

\section{Österreich}

Eine Studie des Ludwig Boltzmann Instituts für Health Technology Assessment stellte 2015 fest, dass Pharmafirmen österreichische Patientinnen-Initiativen mit über einer Million Euro unterstützt haben (Wild et al. 2019). Dafür wurden alle Websites der Mitglieder des Verbands der pharmazeutischen Industrie (PHARMIG) analysiert und komplementär die der Betroffenen-Organisationen. Dies war nur bedingt möglich, da nicht alle über aktuelle oder funktionierende Websites verfügten. Auch auf Seiten der PHARMIG war die Datenlage nicht optimal: Nur 20 Prozent der PHARMIG-Mitgliedsunternehmen $(n=24$ von $\mathrm{n}=115$ ) deklarierten die Zahlungen an solche Gruppierungen. Da sich auf den Websites der Patientinnen-Organisationen aber deutlich mehr Firmenlogos von PHARMIG-Unternehmen fanden, ist von einem „Underreporting“ auszugehen (ebd.). Darüber hinaus wurden nur die direkten Zahlungen erfasst - nicht aber indirektes Sponsoring über Stiftungen, PR-Agenturen oder Verlage. Das passt gut zu den Erfahrungen aus anderen Ländern. Von den insgesamt 1.145.000 Euro gingen über 60 Prozent an die Bereiche Neurologie, Hämato-Onkologie, Rheumatologie und Hämophilie. Den größten Betrag erhielt 2014 die Österreichische Multiple Sklerose Gesellschaft, gefolgt von der Österreichischen Hämophilie Gesellschaft, Hepatitis Hilfe und der Parkinson Selbsthilfe Österreich (ebd.).

\section{Was tun gegen Astroturfing?}

Dass Astroturfing schädlich ist, sollte unstrittig sein Pharmafirmen dürfen nicht über Behandlungsformen entscheiden. Ihr Ziel ist nicht die optimale medizinische Versorgung der Bevölkerung, sondern das Erwirtschaften von Gewinn. Die Maximallösung wäre natürlich, Zulassungs- 
studien für Medikamente und Behandlungen komplett öffentlich $\mathrm{zu}$ finanzieren und auch die Herstellung der Präparate. Zusätzlich müssten alle entsprechenden Daten öffentlich zugänglich sein und unabhängige Kontrollinstanzen mit einem robusten Mandat existieren. Das dürfte momentan nicht durchsetzbar sein - was aber nicht bedeutet, dass man sich mit Astroturfing im Gesundheitsbereich abfinden muss. Ein erster Schritt bestünde in einer global verpflichtenden Offenlegung sämtlicher Finanzierungsquellen von Patientinnen-Initiativen samt „Conflict of Interest“" Erklärung, ob Finanzmittel von der Pharmaindustrie akzeptiert werden. Darüber hinaus muss auch die Offenlegung bei den Pharmakonzernen strikter durchgesetzt, bzw. Nichtbefolgung sanktioniert werden. Es sollte eine globale Datenbank geben, über die Verbindungen und Finanzierungen auch für Laien nachvollziehbar sind. Das würde allen erleichtern, sich zu informieren und damit auch dem informationsethischen Anspruch gerecht werden, dass öffentlich relevante Informationen auch öffentlich verfügbar sind. Solange derartige Empfehlungen nicht durchgesetzt sind, besteht die Herausforderung für die Informationswissenschaften darin, den Fokus auf Dokumente als primäre Quelle zu hinterfragen und das Problem strategischer Informationsvorenthaltung stärker zu berücksichtigen. Der sozialen Bedingtheit von Quellen in der Art und Weise ihres (Nicht)Zustandekommens muss mehr Aufmerksamkeit geschenkt werden.

Deskriptoren: Informationsethik, Medizinethik, Wissenschaftsforschung, Medizinforschung, Propaganda, Desinformationsstrategie

\section{Literatur}

Biancu S. et al. (2016): Fundraising Toolkit for Patient Organisations. https://www.eu-patient.eu/globalassets/policy/funding/epf_ broch2016_v5blow.pdf [8.9.2020].

Boseley S. (18.10.2006): Concern over cancer group's link to drug firm. The Guardian. https://www.theguardian.com/society/ 2006/oct/18/cancercare.health [13.9.2020].

DZI (2012): DZI-Stellungnahme Europäisches Spendensiegel, http:// www.dzi.de/wp-content/pdfs_DZI/EuropSpenSieg15Mai2012. pdf [8.9.2020].

EFPIA Patient Think Tank (2017): Working together with Patient Groups. https://www.efpia.eu/media/412524/working-toge ther-with-patient-groups-23102017.pdf [13.5.2020].

Fleischman W., Agrawal S., King M., et al. (2016): Association between payments from manufacturers of pharmaceuticals to physicians and regional prescribing: cross sectional ecological study. BMJ2016;354:i4189. https://doi.org/10.1136/bmj.i4189.

Horwitz S., Higham S., Bennett D., Kornfield M. (6.12.2019): „SELL BABY SELL“: Inside the opioid industry's marketing machine.
The Washington Post. https://www.washingtonpost.com/gra phics/2019/investigations/opioid-marketing [20.20.2020].

Lorenzo A. (17.7.2018): Treasury defends move to halt nonprofit disclosures amid Wyden threat. Politico. https://www.politico. com/story/2018/07/17/treasury-nonprofit-donor-disclosures690200 [13.9.2020]

Lupkin S. et al. (4.3.2019): Big Pharma Gave Money To Patient Advocacy Groups Opposing Medicare Changes. https://khn.org/ne ws/big-pharma-gave-money-to-patient-advocacy-groups-oppos ing-medicare-changes/ [13.5.2020].

Lurie J. (29.8.2019): Unsealed Documents Show How Purdue Pharma Created a "Pain Movement". https://www.motherjones.com/ crime-justice/2019/08/unsealed-documents-show-how-pur due-pharma-created-a-pain-movement/ [13.5.2020].

McCoy M. S. et al. (2017): Conflicts of Interest for Patient-Advocacy Organizations, in: New England Journal of Medicine, 376 (9): 880-885 doi: 10.1056/NEJMsr1610625 [13.5.2020].

Oreskes N., Conway E. M. (2010): Merchants of Doubt: How a Handful of Scientists Obscured the Truth on Issues from Tobacco Smoke to Global Warming. New York :Bloomsbury Press.

Ozieranski P. Et al. (2019): Exposing drug industry funding of UK patient organisations. British Medical Journal 365:1806 doi: https://doi.org/10.1136/bmj.l1806.

Perehudoff S. K., \& Alves, T. L. (2011): The patient \& consumer voice and pharmaceutical industrysponsorship. Amsterdam: Health Action International. http://hdl.handle.net/11370/d2b12360036b-4eba-894d-f111282ddd30 [13.5.2020].

Perrone M. et al. (15.12.2016): Pro-painkiller echo chamber shaped policy amid drug epidemic. https://publicintegrity.org/statepolitics/pro-painkiller-echo-chamber-shaped-policy-amid-drugepidemic/ [13.5.2020].

Ruoff A. (8.10.2019): AbbVie, Bristol-Myers Among Patient Advocacy Groups' Big Backers. https://about.bgov.com/news/abbvie-bri stol-myers-among-patient-advocacy-groups-big-backers/ [13.5.2020]

Sismondo S. (2018): Ghost-Managed Medicine. Big Pharma's Invisible Hands. Mattering Press.

United States. General Accounting Office (December 19, 2003): Prescription Drugs: OxyContin Abuse and Diversion and Efforts to Address the Problem, report. Washington D.C., https://www. gao.gov/new.items/d04110.pdf [13.9.2020].

Wild C., et al. (2015): Sponsoring von PatientInneninitiativen in Österreich. SystematischeAnalyse. Rapid Assessment 007 b. http://eprints.aihta.at/1072/ [13.9.2020].

Claus Harringer, B. A., M. A.

Johannes Kepler Universität Linz

Institut für Philosophie und Wissenschaftstheorie

Altenberger Straße 69

4040 Linz

claus.harringer@jku.at

https://orcid.org/0000-0001-7697-1311

Claus Harringer ist Mitglied im Vorstand der Österreichischen Gesellschaft für Dokumentation und Information (ÖGDI) und im Fachausschuss „Informationsethik und Wissenschaftsintegrität“ in der ÖGDI. 